\title{
Early potato production is positively affected by potassium fertilization
}

\author{
Ibrahim N. Nasef and Eltohamy A. A. Yousef \\ Department of Horticulture, Faculty of Agriculture, Suez Canal University, Ismailia, Egypt
}

Received: $17 / 2 / 2019$

\begin{abstract}
Two field experiments were conducted during 2017-2018 and 2018-2019, at the Experimental Farm, Faculty of Agriculture, Suez Canal University, Ismailia, Egypt with aim of investigation of potassium application on earliness of potato. To achieve this goal, five different net potassium levels: $0,60,90,120$, and $150 \mathrm{~kg} / \mathrm{Feddan}$ were applied and potato tubers were harvested at two harvesting times: 90 and 105 days after planation. The results reported that both potassium application and harvesting time as well as their interaction significantly affected most of the studied traits. The highest values of plant height, plant fresh weight, plant dry matter, tuber dry matter, marketable yield/feddan, total yield/feddan, soluble solid content (SSC), nitrogen $(\mathrm{N})$ content and potassium $(\mathrm{K})$ content were obtained from the plants received $120 \mathrm{~kg} \mathrm{~K}$ and harvested at 105 days after planation. While, the potassium fertilized plant at 120 $\mathrm{kg} /$ feddan and harvested at 90 days achieved the maximum values of total yield per plant, total sugar and non-reducing sugars. However, the difference between these two treatments was not necessarily significant for some traits. In addition, the results of the current study interestingly clarified that potassium level $(150 \mathrm{~kg})$ negatively affected some yield attributes in both harvesting times compared to the other potassium levels. Consequently, the result of the current study indicates that high level of potassium $(120 \mathrm{~kg})$ can hasten the earliness of potato, which may achieve notable financial profits for potato growers.
\end{abstract}

Keywords: Solanum tuberosum, potassium sulphate, yield, harvesting time

\section{INTRODUCTION}

According to Food and Agriculture Organization of the United Nations (FAO 2017), the global total production of potato tubers was estimated at 388,190,674 tonnes in 2017; while the Egyptian potato production for the same year was estimated at $4,325,478$ tonnes, which represents roughly $1 \%$ of the global production. Therefore, potato is one of the main important solanaceous vegetables grown in Egypt as a leading exportable and cash vegetable crop. From nutritional point of view, potato is a major inexpensive energy source, where contains carbohydrates, fiber, protein, minerals and have low fat (Navarre et al., 2009), consequently it is used in several economic industries, such as chips and French fries as well as alcohol and starch production.

Plant nutrition management can strongly affect the quantity and quality of agricultural products, including potato. Potassium is one of the necessary nutrients for all crop species in order to grow well, since it plays vital roles in plant cell (Mengel and Kirkby, 2001). Likewise, potassium is an essential nutrient for potato growth and production (Dampney et al., 2011); whereas it is absorbed in larger amount compared to other nutrient (Havlin et al., 2005). It is documented that potassium is nearly needed in all physiological processes in potato plants, such as stomata regulation, increasing enzyme activity, improving synthesis of protein, carbohydrates and fats, translocation of photosynthetates (Mueller-Roeber et al., 1995; Abd El-Latif et al., 2011) as a key factor to ensure successful production and growth. Also, it is essential in the activation of enzymes, in meristematic tissues at the growing tips, involved in starch synthesis, $\mathrm{N}$ metabolism, and respiration (Havlin et al., 2005).

It is well documented that potato is heavily potassium fertilized plant since it takes a part in all outgoing physiological process. Bishwoyog and Swarnima (2016) reviewed that a yield of 30 tons of potato tubers will removes $250 \mathrm{~kg}$ of potassium per hector. It has been established in several previous studies that addition of potassium at adequate level, which depends on soil fertility, crop rotation and soil type, improves tuber yield attributes (Zelelew et al., 2016; Haddad et al., 2016), tuber quality attributes (Abd El-Latif et al., 2011; Michalska et al., 2016), plant tolerance to biotic and abiotic stresses (Abd ElLatif et al., 2011; Oyarburo et al., 2015; Liljeroth et al., 2016).

From the economic point of view, earliness trait is very important key in agriculture sector because the early maturity of vegetables offers the advantage of higher prices in the local and international marketplaces. In this context, it was reported that potassium has an effect on early yield of some agricultural crops. For instance, Cutcliffe and Munro (1976) reported a slight early season advantage from high rate of potassium. One the other hand, Pettigrew (2003) and Amanullah et al. (2016) stated that the earlier crop maturity of cotton and maize was associated with low dose of potassium. While, Abay and Sheleme (2011) confirmed that potassium application has no effect on physiological maturity of potato. Nevertheless, the information about the effect of potassium application on early maturity and earliness in potato is limited. Based on the previous information, our hypothesis was to investigate whether high or low dose of potassium hasten the early mature yield in potato. Thus, the aim of the current study was to evaluate the yield and bio-chemical changes in potato tubers at two harvesting times: 90 and 105 days after planting, in response to $0,60,90,120$ and $150 \mathrm{~kg}$ net $\mathrm{K} /$ Feddan. 


\section{MATERIALS AND METHODS}

The experiment was conducted at Research Experimental Farm of the Faculty of Agriculture, Suez Canal University, Ismailia, Egypt, during the two consecutive winter seasons of 2017-2018 and 20182019 to study the effect of potassium fertilization, harvesting time and their interaction on vegetative growth, yield and chemical components of potato Solanum tuberosum L. "simga", which is second early cultivars (105-110 days).

Soil texture was sandy $(85.20 \%$ sand, $11.5 \%$ silt and $3.3 \%$ clay), with $\mathrm{pH} 8.27$, electrical conductivity $0.47 \mathrm{dSm}^{-1}$, calcium $0.4 \mathrm{mM}$, magnesium $0.3 \mathrm{mM}$, potassium $0.3 \mathrm{mM}$, Na $3.0 \mathrm{mM}$, bicarbonate $1.6 \mathrm{mM}$, chloride $3.0 \mathrm{mM}$, and sulfate $0.05 \mathrm{mM}$, available nitrogen $59 \mathrm{ppm}$, available phosphorus $27.5 \mathrm{ppm}$, available potassium $68 \mathrm{ppm}$,

Experimental soil was cleared, ploughed and harrowed, and then drip irrigation system was placed. The organic manure at the rate of $20 \mathrm{~m}^{3} / \mathrm{fad}$. and 400 $\mathrm{kg} / \mathrm{Fed}$. of calcium superphosphate $15.5 \% \mathrm{P}_{2} \mathrm{O}_{5}$ were added at soil preparation. Uniform, healthy and well sprout simga variety of potato tubers were planted at spacing of between rows and plants $80 \mathrm{~cm}$ and $40 \mathrm{~cm}$, respectively. The planting was carried out by hand on $13^{\text {rd }}$ and $18^{\text {th }}$ of October in the first and second season, respectively. The sowing depth was $12-13 \mathrm{~cm}$ and planting was done by placing a tuber manually per hill.

Experimental unit area (plot) was $2.4 \mathrm{~m} \mathrm{x} 4.5 \mathrm{~m}$ in size (three rows). A distance of 1 meter was maintained between plots and 1.5 meters between the blocks.

The experiment was consisting of two factors, the first factor was potassium fertilizer with five levels $0,60,90,120$ and $150 \mathrm{~kg} /$ Feddan and the second one was harvesting time 90 and 105 days after planting. Potassium sulfate $\left(48 \% \mathrm{~K}_{2} \mathrm{O}\right)$ was used as a source of potassium. Potassium was applied four times, the first dose was added after two weeks from sprouting of tubers and the rest doses were supplied with 2 weeks intervals and stopped at age of 75 days. Ammonium nitrate $(33.5 \% \mathrm{~N})$ was used at $300 \mathrm{~kg} /$ Feddan, as source for nitrogen. Cultural practice for potato such as weeding, cultivation and ridging were practiced per recommendation.

\section{Measurements}

\section{Growth parameters}

Nine plants from each replicate were harvested at each harvest times (90 and 105 days). Plant height $(\mathrm{cm})$ was measured using a ruler starting from the base of the main shoot to the apex. Shoot fresh weight (g/plant) was estimated with gravimetric method. To determine, dry matter, N, P and K content of tubers from five randomly selected plants per plot were taken at harvest washed, chopped and mixed. The sample was weighed while fresh and late dried in an oven at $70^{\circ} \mathrm{C}$ for 72 hours and reweighted. It was calculated as the ratio between dry and fresh mass expressed as a percentage. Furthermore the dried sample ground and sieved then $\mathrm{N}, \mathrm{P}$ and $\mathrm{K}$ content of the tuber was determined.

\section{Yield}

Plants of each experimental plot were harvested at two harvest times (90 and 105 days), weighted and yield per plant (g/plant), marketable yield per Feddan (tonnes/Fed), nonmarketable yield per Feddan (tonnes/Fed) and total yield per Feddan (tonnes/Fed) were calculated. Tubers with diameter of bigger than 5 $\mathrm{cm}$ and less than $5 \mathrm{~cm}$ were graded as marketable and non-marketable, respectively.

\section{Chemical composition \\ Organic compounds}

- Soluble solid content (SSC, \%) in the fresh tubers sap was measured by a digital refractometer (Atago N1, Japan).

- Total sugars (mg/g FW) were determined according to Dubois et al. (1956). Reducing and non-reducing sugars were determined according to Sadasivam and Manickam (1991).

- Total phenolic (mg/100g FW) were determined according to Mazumdar and Majumder (2003).

\section{Minerals determination}

$0.5 \mathrm{~g}$ of fine ground tubers was digested with a mixture of sulfuric acid and hydrogen peroxide and then brought to a final volume of $100 \mathrm{ml}$ with distilled water to determine nitrogen, phosphorus, potassium.

- Nitrogen was measured at wavelength of $650 \mathrm{~nm}$ using a spectrophotometer (UNICO UV/Visible 2100 , USA) according to method of Baethgen and Ally (1989).

- Phosphorus: P was analyzed by chlorostannus reduced molybdophosphoric blue color method, in sulfuric acid system at $660 \mathrm{~nm}$ using a spectrophotometer (UNICO UV/Visible 2100, USA) as described by Jackson (1973).

- Potassium was determined using a Perkin-elmer, Flame photometer (Page, 1982).

\section{Statistical analysis}

The experiments were organized in a completely randomized block design (CRBD) with a split plot arrangement, with three replications, in which each replication considered as a block. Potassium fertilizer was randomly distributed in the main plots and harvest time treatments were randomly distributed in the subplots. Data were analyzed by two-way analysis of variance (ANOVA) using CoStat version 6.303 19982004 CoHort software, 798 Lighthouse Ave, PMP 320, Monterey, CA 93940, USA. Duncan's test used to compare means at the $1 \%$ significance level.

\section{RESULTS}

Effect of harvesting time, potassium levels and their interaction on vegetative parameters

The data presented in Table (1) shows the main effect of harvesting time, potassium levels and their 
interaction on the vegetative growth in growing seasons: 2016/2017 and 2017/2018. It clearly shows that there were highly significant differences between the two harvesting times in all studied traits with exception of tuber dry matter in season of 2017/2018. It also, show that there were highly significant differences among the potassium levels and resulted in a significant increase in all traits under the study compared to control; whereas all traits increased to their maximum with potassium level of $120 \mathrm{~kg} /$ Feddan in both seasons. Regarding the interaction between the harvesting time and potassium level, Table (1) show that the potato plants which harvested at 105 days and received $120 \mathrm{~kg} /$ Feddan gave the highest values for all traits in both season followed by plants that harvested at 90 days and got $120 \mathrm{~kg}$ and/or plants that harvested at 105 days and got $90 \mathrm{~kg} /$ Feddan. However, the differences between these treatments were not necessarily significant.

Table (1): Main effects of potassium levels, harvesting time and their interactions on vegetative growth of potato during 2017/2018 and 2018/2019 seasons

\begin{tabular}{|c|c|c|c|c|c|c|c|}
\hline \multirow{3}{*}{ Parameters } & \multirow{3}{*}{ K level } & \multicolumn{3}{|c|}{$2017 / 2018$} & \multicolumn{3}{|c|}{ 2018/2019 } \\
\hline & & \multicolumn{2}{|c|}{ Harvesting time } & \multirow{2}{*}{ Mean } & \multicolumn{2}{|c|}{ Harvesting time } & \multirow{2}{*}{ Mean } \\
\hline & & 90 days & 105 days & & 90 days & 105 days & \\
\hline \multirow{6}{*}{$\begin{array}{l}\text { Plant height } \\
\text { (cm) }\end{array}$} & $0 \mathrm{~kg}$ & $35.33 d$ & $43.67 \mathrm{c}$ & 39.50D & $38.17 \mathrm{e}$ & $43.33 d$ & 40.75D \\
\hline & $60 \mathrm{~kg}$ & $43.67 \mathrm{c}$ & $47.00 \mathrm{c}$ & $45.33 \mathrm{C}$ & $43.00 \mathrm{~d}$ & $48.67 \mathrm{c}$ & $45.83 C$ \\
\hline & $90 \mathrm{~kg}$ & $46.00 \mathrm{c}$ & $52.67 \mathrm{~b}$ & 49.33B & $48.00 \mathrm{~cd}$ & $54.33 b$ & 51.17B \\
\hline & $120 \mathrm{~kg}$ & $53.00 \mathrm{~b}$ & $59.67 \mathrm{a}$ & $56.33 \mathrm{~A}$ & $55.33 b$ & $61.67 \mathrm{a}$ & $\mathbf{5 8 . 5 0 A}$ \\
\hline & $150 \mathrm{~kg}$ & $44.00 \mathrm{c}$ & $45.67 \mathrm{c}$ & 44.83C & $45.00 \mathrm{~cd}$ & $44.00 \mathrm{~cd}$ & 44.50CD \\
\hline & Mean & 44.40B & $49.73 \mathrm{~A}$ & & 45.90B & $50.40 \mathrm{~A}$ & \\
\hline \multirow{6}{*}{$\begin{array}{l}\text { Fresh weight } \\
\text { (g / plant) }\end{array}$} & $0 \mathrm{~kg}$ & $123.16 \mathrm{c}$ & $140.09 \mathrm{c}$ & 131.62D & $127.28 \mathrm{c}$ & $139.78 \mathrm{c}$ & 133.53D \\
\hline & $60 \mathrm{~kg}$ & $227.1 \mathrm{~b}$ & $242.43 b$ & 234.77C & $230.27 b$ & $246.89 b$ & 238.58C \\
\hline & $90 \mathrm{~kg}$ & $238.1 b$ & $297.49 a$ & 267.8B & $242.83 b$ & $303.17 \mathrm{a}$ & 273.00B \\
\hline & $120 \mathrm{~kg}$ & $306.25 \mathrm{a}$ & $318.96 \mathrm{a}$ & $312.61 \mathrm{~A}$ & $312.68 \mathrm{a}$ & $326.69 a$ & $319.68 A$ \\
\hline & $150 \mathrm{~kg}$ & $216.9 \mathrm{~b}$ & $231.83 b$ & 224.37C & $220.90 \mathrm{~b}$ & $228.69 \mathrm{~b}$ & $224.80 \mathrm{C}$ \\
\hline & Mean & $222.30 \mathrm{~B}$ & $246.16 A$ & & 226.79B & $249.04 A$ & \\
\hline \multirow{6}{*}{$\begin{array}{l}\text { Plant dry matter } \\
\qquad(\%)\end{array}$} & $0 \mathrm{~kg}$ & $10.67 \mathrm{de}$ & $12.25 \mathrm{bc}$ & 11.46B & $11.81 \mathrm{f}$ & $13.92 \mathrm{~cd}$ & $12.87 \mathrm{C}$ \\
\hline & $60 \mathrm{~kg}$ & $11.81 \mathrm{bcd}$ & $12.25 \mathrm{bc}$ & $12.03 \mathrm{AB}$ & $13.45 \mathrm{de}$ & $14.94 \mathrm{bc}$ & 14.2B \\
\hline & $90 \mathrm{~kg}$ & $10.79 \mathrm{de}$ & $13.76 \mathrm{a}$ & $12.28 \mathrm{~A}$ & $12.23 \mathrm{ef}$ & $15.87 \mathrm{ab}$ & $14.05 B$ \\
\hline & $120 \mathrm{~kg}$ & $11.91 \mathrm{bcd}$ & $13.01 \mathrm{ab}$ & $12.46 \mathrm{~A}$ & $15.81 \mathrm{ab}$ & $16.46 \mathrm{a}$ & $16.14 \mathrm{~A}$ \\
\hline & $150 \mathrm{~kg}$ & $9.52 \mathrm{e}$ & $11.51 \mathrm{~cd}$ & $10.52 \mathrm{C}$ & $11.63 \mathrm{f}$ & $14.41 \mathrm{~cd}$ & 13.02C \\
\hline & Mean & $10.94 B$ & $12.56 \mathrm{~A}$ & & $12.98 B$ & $15.12 \mathrm{~A}$ & \\
\hline \multirow{6}{*}{$\begin{array}{l}\text { Tuber dry matter } \\
(\%)\end{array}$} & $0 \mathrm{~kg}$ & $14.40 \mathrm{~d}$ & $12.22 \mathrm{e}$ & 13.31B & $13.00 \mathrm{e}$ & $13.43 \mathrm{e}$ & $13.22 \mathrm{C}$ \\
\hline & $60 \mathrm{~kg}$ & $15.55 \mathrm{bcd}$ & 16.02a-d & $15.79 \mathrm{~A}$ & $17.03 \mathrm{~cd}$ & $18.13 \mathrm{bcd}$ & 17.58B \\
\hline & $90 \mathrm{~kg}$ & 15.96a-d & 17.48ab & $16.72 \mathrm{~A}$ & $17.87 \mathrm{~cd}$ & $20.21 \mathrm{ab}$ & $19.04 \mathrm{AB}$ \\
\hline & $120 \mathrm{~kg}$ & $16.74 \mathrm{abc}$ & $17.87 \mathrm{a}$ & $17.31 \mathrm{~A}$ & $18.74 \mathrm{bc}$ & $21.51 \mathrm{a}$ & 20.13A \\
\hline & $150 \mathrm{~kg}$ & $15.18 \mathrm{~cd}$ & $16.21 \mathrm{a}-\mathrm{d}$ & 15.69A & $16.46 \mathrm{~d}$ & $17.97 \mathrm{~cd}$ & 17.22B \\
\hline & Mean & $15.56 \mathrm{~A}$ & $15.96 \mathrm{~A}$ & & $16.62 B$ & $18.25 \mathrm{~A}$ & \\
\hline
\end{tabular}

Values are the means of three replicates. Values followed by the same letters within a column for each genus are not significantly different at the $1 \%$ level of probability according to Duncan's multiple range test 
Effect of harvesting time, potassium level and their interaction on yield and yield components

Table (2) shows that no significant differences between the two harvesting times were observed, however they were observed among potassium levels in terms of yield and yield components. The results indicated that the application of potassium at 120 $\mathrm{kg} /$ Feddan was more effective than control and other potassium levels for enhancing total yield/plant, marketable yield and total yield per Feddan as well as decreasing the non-marketable yield. Additionally, the interaction effects between harvesting time and potassium level are presented in Table (2). It interestingly shows that potato plants that were harvested at 90 days and supplemented with potassium at $120 \mathrm{~kg} / \mathrm{Feddan}$ had the highest total yield per plant and total yield per Feddan in both seasons. Statistically, the same values of total yield, marketable yield and yield per plant were found in plants received $90 \mathrm{~kg} /$ fed and harvested after 105 days. It is clear that the late harvesting time (105 days) in the plants received 120 $\mathrm{kg} /$ feddan did not improve the yield. However, the plants received $90 \mathrm{~kg} /$ feddan needed more time to reach the maturity stage (105).

Table (2): Main effects of potassium levels, harvesting time and their interactions on yield and yield components of potato during 2017/2018 and 2018/2019 seasons

\begin{tabular}{|c|c|c|c|c|c|c|c|}
\hline \multirow{3}{*}{ Parameters } & \multirow{3}{*}{ K level } & \multicolumn{3}{|c|}{$2017 / 2018$} & \multicolumn{3}{|c|}{ 2018/2019 } \\
\hline & & \multicolumn{2}{|c|}{ Harvesting time } & \multirow{2}{*}{ Mean } & \multicolumn{2}{|c|}{ Harvesting time } & \multirow{2}{*}{ Mean } \\
\hline & & 90 days & 105 days & & 90 days & 105 days & \\
\hline \multirow{6}{*}{$\begin{array}{c}\text { Yield/Plant } \\
\text { (g) }\end{array}$} & $0 \mathrm{~kg}$ & $340.93 \mathrm{e}$ & $429.85 \mathrm{e}$ & 385.39C & $436.03 f$ & $554.59 f$ & 495.31C \\
\hline & $60 \mathrm{~kg}$ & $674.94 d$ & $760.51 \mathrm{~cd}$ & 717.72B & $761.53 \mathrm{de}$ & $832.03 \mathrm{~cd}$ & 796.78B \\
\hline & $90 \mathrm{~kg}$ & $845.16 b c$ & $920.39 \mathrm{ab}$ & $882.78 A$ & $937.84 b c$ & $1013.05 \mathrm{ab}$ & 975.45A \\
\hline & $120 \mathrm{~kg}$ & $1007.57 \mathrm{a}$ & $981.21 \mathrm{ab}$ & 994.39A & $1089.54 \mathrm{a}$ & $1078.13 \mathrm{a}$ & $1083.84 A$ \\
\hline & $150 \mathrm{~kg}$ & $713.55 \mathrm{~cd}$ & $622.28 \mathrm{~d}$ & 667.92B & $793.2 \mathrm{de}$ & $692.31 \mathrm{e}$ & 742.76B \\
\hline & Mean & 716.43A & 742.84A & & $803.63 A$ & 834.02A & \\
\hline \multirow{6}{*}{$\begin{array}{c}\text { Marketable } \\
\text { yield } \\
\text { (Ton / Feddan) }\end{array}$} & $0 \mathrm{~kg}$ & $3.82 \mathrm{~d}$ & $5.09 \mathrm{~d}$ & $4.46 C$ & $4.42 \mathrm{~d}$ & $5.78 \mathrm{~d}$ & $5.10 \mathrm{C}$ \\
\hline & $60 \mathrm{~kg}$ & $8.04 \mathrm{c}$ & $9.33 \mathrm{bc}$ & 8.69B & $8.74 \mathrm{c}$ & $10.07 \mathrm{c}$ & $9.40 B$ \\
\hline & $90 \mathrm{~kg}$ & $10.9 \mathrm{ab}$ & $11.18 \mathrm{a}$ & $11.04 A$ & $11.8 \mathrm{~b}$ & $12.12 \mathrm{ab}$ & $11.96 \mathrm{~A}$ \\
\hline & $120 \mathrm{~kg}$ & $12.68 \mathrm{a}$ & $12.71 \mathrm{a}$ & $12.70 \mathrm{~A}$ & $13.54 \mathrm{ab}$ & $13.79 \mathrm{a}$ & $13.66 \mathrm{~A}$ \\
\hline & $150 \mathrm{~kg}$ & $8.84 \mathrm{c}$ & $7.79 \mathrm{c}$ & 8.31B & $9.53 \mathrm{c}$ & $8.43 c$ & 8.98B \\
\hline & Mean & $8.86 \mathrm{~A}$ & $9.22 \mathrm{~A}$ & & $9.61 \mathrm{~A}$ & $10.04 \mathrm{~A}$ & \\
\hline \multirow{6}{*}{$\begin{array}{c}\text { Non-marketable } \\
\text { yield } \\
\text { (Ton / Feddan) }\end{array}$} & $0 \mathrm{~kg}$ & $0.65 \mathrm{ab}$ & $0.55 \mathrm{ab}$ & $0.60 \mathrm{AB}$ & $1.31 \mathrm{ab}$ & $1.50 \mathrm{a}$ & $1.40 \mathrm{~A}$ \\
\hline & $60 \mathrm{~kg}$ & $0.82 \mathrm{a}$ & $0.65 \mathrm{ab}$ & $0.73 \mathrm{~A}$ & $1.26 \mathrm{ab}$ & $0.86 \mathrm{bcd}$ & $1.06 B$ \\
\hline & $90 \mathrm{~kg}$ & $0.19 b$ & $0.90 \mathrm{a}$ & $0.54 \mathrm{ABC}$ & $0.51 \mathrm{~d}$ & $1.18 \mathrm{abc}$ & $0.85 C$ \\
\hline & $120 \mathrm{~kg}$ & $0.55 \mathrm{ab}$ & $0.17 b$ & $0.36 \mathrm{C}$ & $0.76 \mathrm{bcd}$ & $0.36 \mathrm{~d}$ & $0.56 \mathrm{D}$ \\
\hline & $150 \mathrm{~kg}$ & $0.53 \mathrm{ab}$ & $0.37 \mathrm{ab}$ & $0.45 B C$ & $0.88 \mathrm{bcd}$ & $0.65 \mathrm{~cd}$ & 0.77CD \\
\hline & Mean & $0.53 \mathrm{~A}$ & $0.55 \mathrm{~A}$ & & $0.91 \mathrm{~A}$ & $0.94 \mathrm{~A}$ & \\
\hline \multirow{6}{*}{$\begin{array}{c}\text { Total yield } \\
\text { (Ton / Feddan) }\end{array}$} & $0 \mathrm{~kg}$ & $4.47 \mathrm{e}$ & $5.64 \mathrm{e}$ & $5.06 \mathrm{C}$ & $5.72 \mathrm{f}$ & $7.28 \mathrm{f}$ & $6.50 \mathrm{C}$ \\
\hline & $60 \mathrm{~kg}$ & $8.86 \mathrm{~d}$ & $9.98 \mathrm{~cd}$ & 9.42B & 10.00de & $10.92 \mathrm{~cd}$ & $10.46 \mathrm{~B}$ \\
\hline & $90 \mathrm{~kg}$ & $11.09 \mathrm{bc}$ & $12.08 \mathrm{ab}$ & 11.59A & $12.31 b c$ & $13.3 \mathrm{ab}$ & $12.80 \mathrm{~A}$ \\
\hline & $120 \mathrm{~kg}$ & $13.22 \mathrm{a}$ & $12.88 \mathrm{ab}$ & $13.05 \mathrm{~A}$ & $14.30 \mathrm{a}$ & $14.15 \mathrm{a}$ & $14.23 \mathrm{~A}$ \\
\hline & $150 \mathrm{~kg}$ & $9.37 \mathrm{~cd}$ & $8.17 \mathrm{~d}$ & 8.77B & $10.41 \mathrm{de}$ & $9.09 \mathrm{e}$ & $9.75 \mathrm{~B}$ \\
\hline & Mean & $9.40 \mathrm{~A}$ & $9.74 \mathrm{~A}$ & & $10.55 A$ & $10.95 A$ & \\
\hline
\end{tabular}

Values are the means of three replicates. Values followed by the same letters within a column for each genus are not significantly different at the $1 \%$ level of probability according to Duncan's multiple range test 
Effect of harvesting time, potassium level and their interaction on organic compounds

In season of $2017 / 2018$, only SSC and total phenolic show significant differences between harvesting times, however all organic compounds show significant differences between the two harvesting times in season of 2018/2019 with the exception of the total sugar content (Table 3). Also, the main effect of potassium level significantly influenced all organic compounds in both growing seasons (Table 3). Where, potassium addition at level of $120 \mathrm{~kg}$ recorded highest values of total sugars and non-reducing sugars as well as lowest values of reducing sugars and total phenolic in both growing seasons. Regarding the interaction, Table (3) shows that generally the highest total sugars content and non-reducing sugars were observed in plants fertilized with $120 \mathrm{~kg} /$ Feddan and early harvested at age of 90 days. Also, this treatment achieved the lowest values of reducing sugars and total phenolic (Table 3). The results reported that the highest values of SSC content were recorded with the combination of $120 \mathrm{~kg} / \mathrm{Feddan}$ and harvesting at 105 days after plantation.

Table (3): Main effects of potassium levels, harvesting time and their interactions on organic compounds of potato tubers during 2017/2018 and 2018/2019 seasons.

\begin{tabular}{|c|c|c|c|c|c|c|c|}
\hline \multirow{3}{*}{ Parameters } & \multirow{3}{*}{ K level } & \multicolumn{3}{|c|}{$2017 / 2018$} & \multicolumn{3}{|c|}{$2018 / 2019$} \\
\hline & & \multicolumn{2}{|c|}{ Harvesting time } & \multirow{2}{*}{ Mean } & \multicolumn{2}{|c|}{ Harvesting time } & \multirow{2}{*}{ Mean } \\
\hline & & 90 days & 105 days & & 90 days & 105 days & \\
\hline \multirow{6}{*}{$\begin{array}{l}\text { SSC } \\
(\%)\end{array}$} & $0 \mathrm{~kg}$ & $2.73 \mathrm{e}$ & $3.13 \mathrm{cde}$ & 2.93B & $3.07 \mathrm{e}$ & $3.33 \mathrm{cde}$ & $3.20 \mathrm{~B}$ \\
\hline & $60 \mathrm{~kg}$ & $3.07 \mathrm{de}$ & $3.73 \mathrm{a}$ & $3.40 \mathrm{~A}$ & $3.23 \mathrm{de}$ & $3.93 \mathrm{ab}$ & $3.58 \mathrm{AB}$ \\
\hline & $90 \mathrm{~kg}$ & $3.53 \mathrm{abc}$ & $3.5 \mathrm{a}-\mathrm{d}$ & $3.52 \mathrm{~A}$ & $3.77 \mathrm{a}-\mathrm{d}$ & $3.87 \mathrm{abc}$ & $3.82 \mathrm{~A}$ \\
\hline & $120 \mathrm{~kg}$ & $3.60 \mathrm{ab}$ & $3.80 \mathrm{a}$ & $3.70 \mathrm{~A}$ & $3.83 \mathrm{abc}$ & $4.20 \mathrm{a}$ & $4.02 \mathrm{~A}$ \\
\hline & $150 \mathrm{~kg}$ & $3.27 \mathrm{bcd}$ & $3.67 \mathrm{ab}$ & $3.47 \mathrm{~A}$ & $3.53 b-e$ & $3.87 \mathrm{abc}$ & $3.70 \mathrm{~A}$ \\
\hline & Mean & $3.24 \mathrm{~B}$ & $3.57 \mathrm{~A}$ & & $3.49 \mathrm{~B}$ & $3.84 \mathrm{~A}$ & \\
\hline \multirow{6}{*}{$\begin{array}{l}\text { Total Sugars } \\
(\mathrm{mg} / \mathrm{g})\end{array}$} & $0 \mathrm{~kg}$ & $3.11 \mathrm{e}$ & $7.11 \mathrm{~d}$ & $5.11 \mathrm{C}$ & $4.53 \mathrm{e}$ & $9.45 \mathrm{~d}$ & 6.99D \\
\hline & $60 \mathrm{~kg}$ & $10.27 \mathrm{bc}$ & $9.25 \mathrm{bcd}$ & 9.76AB & $13.16 b c$ & $11.51 \mathrm{~cd}$ & 12.34B \\
\hline & $90 \mathrm{~kg}$ & $10.52 b$ & $8.16 \mathrm{~cd}$ & 9.34B & $13.85 \mathrm{ab}$ & $10.53 d$ & 12.19B \\
\hline & $120 \mathrm{~kg}$ & $12.82 \mathrm{a}$ & $11 \mathrm{ab}$ & $11.91 \mathrm{~A}$ & $16.04 \mathrm{a}$ & $13.96 \mathrm{ab}$ & $15.00 \mathrm{~A}$ \\
\hline & $150 \mathrm{~kg}$ & $7.90 \mathrm{~d}$ & $8.00 \mathrm{~cd}$ & 7.95B & $9.79 d$ & $9.66 \mathrm{~d}$ & $9.73 C$ \\
\hline & Mean & $8.92 \mathrm{~A}$ & $8.70 \mathrm{~A}$ & & $11.47 \mathrm{~A}$ & $11.02 \mathrm{~A}$ & \\
\hline \multirow{6}{*}{$\begin{array}{c}\text { Reducing Sugars } \\
(\mathrm{mg} / \mathrm{g})\end{array}$} & $0 \mathrm{~kg}$ & $2.27 \mathrm{~b}$ & $3.45 \mathrm{a}$ & $2.86 \mathrm{~A}$ & $4.60 \mathrm{a}$ & $3.30 \mathrm{~b}$ & $3.95 \mathrm{~A}$ \\
\hline & $60 \mathrm{~kg}$ & $0.66 \mathrm{~d}$ & $0.68 \mathrm{~d}$ & $0.67 \mathrm{BC}$ & $1.02 \mathrm{~d}$ & $0.81 \mathrm{~d}$ & $0.91 B$ \\
\hline & $90 \mathrm{~kg}$ & $0.77 \mathrm{~d}$ & $0.61 \mathrm{~d}$ & $0.69 B C$ & $0.77 \mathrm{~d}$ & $0.76 \mathrm{~d}$ & $0.77 B$ \\
\hline & $120 \mathrm{~kg}$ & $0.61 \mathrm{~d}$ & $0.45 \mathrm{~d}$ & $0.53 \mathrm{C}$ & $0.56 \mathrm{~d}$ & $0.62 d$ & $0.59 B$ \\
\hline & $150 \mathrm{~kg}$ & $1.26 \mathrm{~cd}$ & $1.73 b c$ & $1.50 \mathrm{~B}$ & $2.1 \mathrm{c}$ & $1.23 \mathrm{~cd}$ & $1.66 \mathrm{~B}$ \\
\hline & Mean & $1.11 \mathrm{~A}$ & $1.38 \mathrm{~A}$ & & $1.81 \mathrm{~A}$ & $1.34 \mathrm{~B}$ & \\
\hline \multirow{6}{*}{$\begin{array}{l}\text { Non-reducing } \\
\text { sugars } \\
(\mathrm{mg} / \mathrm{g})\end{array}$} & $0 \mathrm{~kg}$ & $0.80 \mathrm{f}$ & $3.47 \mathrm{e}$ & 2.13D & $1.160 \mathrm{~g}$ & $4.60 \mathrm{f}$ & 2.88D \\
\hline & $60 \mathrm{~kg}$ & $9.14 b c$ & $8.13 \mathrm{bcd}$ & 8.63B & $11.74 \mathrm{bc}$ & $9.97 \mathrm{~cd}$ & $10.85 B$ \\
\hline & $90 \mathrm{~kg}$ & $9.26 \mathrm{bc}$ & $7.17 \mathrm{~cd}$ & 8.22BC & $12.44 \mathrm{~b}$ & $9.27 \mathrm{~d}$ & $10.85 B$ \\
\hline & $120 \mathrm{~kg}$ & $11.60 \mathrm{a}$ & $10.02 \mathrm{ab}$ & $10.81 \mathrm{~A}$ & $14.65 \mathrm{a}$ & $12.72 b$ & 13.69A \\
\hline & $150 \mathrm{~kg}$ & $6.31 \mathrm{~d}$ & $6.27 \mathrm{~d}$ & $6.29 \mathrm{C}$ & $8.13 \mathrm{de}$ & $7.19 \mathrm{e}$ & $7.66 \mathrm{C}$ \\
\hline & Mean & $7.42 \mathrm{~A}$ & $7.01 \mathrm{~A}$ & & $9.62 \mathrm{~A}$ & $8.75 B$ & \\
\hline \multirow{6}{*}{$\begin{array}{l}\text { Total Phenolic } \\
\text { (mg/100 g) }\end{array}$} & $0 \mathrm{~kg}$ & $5.86 \mathrm{bc}$ & $9.55 \mathrm{a}$ & $7.70 \mathrm{~A}$ & $7.74 \mathrm{bcd}$ & $11.77 \mathrm{a}$ & $9.76 \mathrm{~A}$ \\
\hline & $60 \mathrm{~kg}$ & $5.26 \mathrm{bc}$ & $8.34 \mathrm{ab}$ & $6.80 \mathrm{AB}$ & $6.87 \mathrm{~cd}$ & $10.8 \mathrm{ab}$ & $8.84 \mathrm{~A}$ \\
\hline & $90 \mathrm{~kg}$ & $5.59 \mathrm{bc}$ & $4.77 b c$ & $5.18 \mathrm{BC}$ & $7.06 \mathrm{~cd}$ & $6.16 \mathrm{~cd}$ & $6.61 B$ \\
\hline & $120 \mathrm{~kg}$ & $3.42 \mathrm{c}$ & $4.94 b c$ & $4.18 C$ & $4.29 \mathrm{~d}$ & $6.24 \mathrm{~cd}$ & 5.27B \\
\hline & $150 \mathrm{~kg}$ & $5.82 \mathrm{bc}$ & $7.44 \mathrm{ab}$ & $6.63 \mathrm{AB}$ & $7.38 \mathrm{bcd}$ & $9.28 \mathrm{abc}$ & $8.33 \mathrm{~A}$ \\
\hline & Mean & 5.19B & $7.00 \mathrm{~A}$ & & 6.67B & $8.85 \mathrm{~A}$ & \\
\hline
\end{tabular}

Values are the means of three replicates. Values followed by the same letters within a column for each genus are not significantly different at the $1 \%$ level of probability according to Duncan's multiple range test 
Effect of harvesting time, potassium level and their interaction on mineral composition

The main effect of harvesting time and potassium level and their interaction on $\mathrm{N}, \mathrm{P}$ and $\mathrm{K}$ are presented in Table (4). Harvesting at age of 105 after plantation recorded high $\mathrm{N}, \mathrm{P}$ and $\mathrm{K}$ content compared to harvesting at 90 days and this difference between two harvesting times was significant only in terms of $\mathrm{K}$ content in season 2017/2018 and in terms of $\mathrm{N}$ and $\mathrm{P}$ in season of 2018/2019. Potassium un-fertilized potato plants, over the two harvesting times, had the statistical highest $\mathrm{N}$ content, while, the lowest $\mathrm{N}$ content was observed in high potassium fertilized $(150 \mathrm{~kg} /$ Feddan $)$ potato plants in both growing seasons (Table 4). In terms of $\mathrm{P}$ and $\mathrm{K}$, the fertilization with $120 \mathrm{~kg}$ potassium per Feddan recorded the high significant values of $\mathrm{P}$ and $\mathrm{K}$ in a comparison to the control and the other potassium levels in growing season. With respect to the interaction effect between harvesting time and potassium treatments, the results showed that potassium un-fertilized potato plants which harvested at late age (105 days) had the highest significant values of $\mathrm{N}$ content in a comparison with other combinations. However, the combination between harvesting at 105 days and potassium fertilization at $120 \mathrm{~kg} /$ Feddan resulted in a highest $\mathrm{K}$ content compared with other combinations and it also achieved the highest $\mathrm{P}$ content but only in 2018/2019 season.

Table (4): Main effects of potassium levels, harvesting time and their interactions on mineral composition of potato during 2017/2018 and 2018/2019 seasons

\begin{tabular}{|c|c|c|c|c|c|c|c|}
\hline \multirow{3}{*}{ Parameters } & \multirow{3}{*}{ K level } & \multicolumn{3}{|c|}{$2017 / 2018$} & \multicolumn{3}{|c|}{ 2018/2019 } \\
\hline & & \multicolumn{2}{|c|}{ Harvesting time } & \multirow{2}{*}{ Mean } & \multicolumn{2}{|c|}{ Harvesting time } & \multirow{2}{*}{ Mean } \\
\hline & & 90 days & 105 days & & 90 days & 105 days & \\
\hline \multirow{6}{*}{$\begin{array}{c}\mathrm{N} \\
(\mathrm{mg} / \mathrm{g} D W)\end{array}$} & $0 \mathrm{~kg}$ & $10.92 \mathrm{abc}$ & $14.59 \mathrm{a}$ & $12.76 \mathrm{~A}$ & $13.72 b c$ & $16.97 \mathrm{a}$ & $15.35 \mathrm{~A}$ \\
\hline & $60 \mathrm{~kg}$ & $9.10 \mathrm{bc}$ & $10.27 \mathrm{bc}$ & $9.69 \mathrm{AB}$ & $11.22 \mathrm{~cd}$ & $13.31 \mathrm{bc}$ & $12.27 \mathrm{~B}$ \\
\hline & $90 \mathrm{~kg}$ & $9.62 b c$ & $9.76 \mathrm{bc}$ & 9.69AB & $11.49 \mathrm{~cd}$ & $12.75 b c$ & 12.12B \\
\hline & $120 \mathrm{~kg}$ & $12.12 \mathrm{ab}$ & $10.31 \mathrm{bc}$ & $A B r^{Y} .11$ & $12.91 \mathrm{bc}$ & $14.20 \mathrm{~b}$ & $13.56 \mathrm{AB}$ \\
\hline & $150 \mathrm{~kg}$ & $7.64 \mathrm{c}$ & $9.40 \mathrm{bc}$ & $8.52 B$ & $7.88 \mathrm{e}$ & $10.00 \mathrm{de}$ & $8.94 \mathrm{C}$ \\
\hline & Mean & $9.88 \mathrm{~A}$ & $\mathbf{A}^{\wedge \vee 10 .}$ & & 11.45B & $13.45 \mathrm{~A}$ & \\
\hline \multirow{6}{*}{$\begin{array}{c}P \\
(\mathrm{mg} / \mathrm{g} D W)\end{array}$} & $0 \mathrm{~kg}$ & $2.46 \mathrm{ab}$ & $2.71 \mathrm{ab}$ & 2.59A & $2.82 \mathrm{f}$ & $3.22 \mathrm{def}$ & $3.02 \mathrm{C}$ \\
\hline & $60 \mathrm{~kg}$ & $2.41 \mathrm{ab}$ & $2.54 \mathrm{ab}$ & $2.47 \mathrm{~A}$ & $3.06 \mathrm{ef}$ & $3.31 \mathrm{def}$ & $3.19 \mathrm{C}$ \\
\hline & $90 \mathrm{~kg}$ & $2.53 \mathrm{ab}$ & $2.32 b$ & $2.43 \mathrm{~A}$ & $3.39 \mathrm{cde}$ & $3.94 b$ & 3.67B \\
\hline & $120 \mathrm{~kg}$ & $2.83 \mathrm{ab}$ & $2.80 \mathrm{ab}$ & $2.82 \mathrm{~A}$ & $3.87 \mathrm{bc}$ & $4.47 \mathrm{a}$ & $4.17 \mathrm{~A}$ \\
\hline & $150 \mathrm{~kg}$ & $2.50 \mathrm{ab}$ & $2.89 \mathrm{a}$ & 2.69A & $3.56 \mathrm{bcde}$ & $3.66 \mathrm{bcd}$ & 3.61B \\
\hline & Mean & $2.54 \mathrm{~A}$ & $2.56 \mathrm{~A}$ & & 3.34B & $3.72 \mathrm{~A}$ & \\
\hline \multirow{6}{*}{$\underset{(\mathrm{mg} / \mathrm{gDW})}{\mathrm{K}}$} & $0 \mathrm{~kg}$ & $44.86 \mathrm{~cd}$ & $44.30 \mathrm{~d}$ & 44.58C & $48.83 \mathrm{~d}$ & $48.09 \mathrm{~d}$ & 48.46C \\
\hline & $60 \mathrm{~kg}$ & $45.25 \mathrm{~cd}$ & $48.39 \mathrm{a}$ & 46.82B & $52.25 \mathrm{c}$ & $51.25 \mathrm{c}$ & 51.75B \\
\hline & $90 \mathrm{~kg}$ & $46.05 \mathrm{bcd}$ & $47.43 \mathrm{ab}$ & 46.74B & $50.79 \mathrm{c}$ & $51.52 \mathrm{c}$ & 51.15B \\
\hline & $120 \mathrm{~kg}$ & $48.62 \mathrm{a}$ & $49.03 a$ & 48.83A & $54.62 b$ & $57.34 \mathrm{a}$ & $55.98 A$ \\
\hline & $150 \mathrm{~kg}$ & $46.34 \mathrm{bc}$ & $47.74 \mathrm{ab}$ & 47.04B & $52.01 \mathrm{c}$ & $51.77 \mathrm{c}$ & 51.89B \\
\hline & Mean & 46.22B & 47.38A & & $51.70 \mathrm{~A}$ & 51.99A & \\
\hline
\end{tabular}

Values are the means of three replicates. Values followed by the same letters within a column for each genus are not significantly different at the $1 \%$ level of probability according to Duncan's multiple range test

\section{DISCUSSION}

Potassium strongly influences potato vegetative growth, tuberization and maturity in different ways. The current research clearly show that vegetative growth traits showed significant differences due to potassium application. All vegetative traits: plant height, plant fresh weight and dry matter of plant as well as tuber showed gradual increases with increasing of potassium level compared to the control, with exception of $150 \mathrm{~kg} /$ feddan. Similarly, several previous studies confirmed the increases in vegetative growth attributes with potassium increment (Mahmoud and Hafez, 2010; Radwan and El-Shall, 2011; Zelelew et al., 2016). This increment in potato vegetative growth might be due to the potassium role in plant nutrition; whereas it is enhancing enzymes activity, protein synthesis and translocations assimilation (Abd El-Latif et al., 2011). In the same regard, this promotional effect potassium might due to existence a synergetic relationship between $\mathrm{K}$ and $\mathrm{N}$ (Saha et al., 2001), which increases the foliage and leaf area index, leading to increasing photosynthesis efficiency. In the same context, potassium has an essential role in plant physiological functions, such as gas exchange, regulation of water, photosynthesis, and translocation of carbohydrate through controlling guard cells movement via turgor regulation Marschner (2012). 
Also, potassium had significant effects on yield traits: total yield per plant, marketable and nonmarketable per Feddan. The maximum tuber yield per plant and marketable as well as the lowest nonmarketable yield were associated with application of $120 \mathrm{~kg}$ potassium, which reflected on total yield per Feddan in both growing season. Similar results were found by Haddad et al. (2016) and Khan et al. (2012). They found a positive relationship between yield traits (marketable yield, tuber weight and total yield) and increasing potassium application. The recorded increases of potassium fertilized potato plants in current experiment might lie on the increased vegetative growth as mentioned above or /and due to the promotional role of potassium in nutrients and water absorption which ultimately improve the total yield (Zelelew et al., 2016). In the same way, potassium promotes movement of photosynthates, amino acids and sucrose to the tubers and enhances loading phloem with sucrose, leading to an increase in rate of solutes of phloem-sap. The significant increases in yield might be also attributed to the potential osmotic of potassium in tubers and its function in ATP synthesis, which gave energy for the photosynthates loading, thus helps potato tubers to have heavier weight and large size. Also, the crucial role of potassium in the stems formation quality may be due to its promotion of synthesis of photosynthates and converted them to starch and protein (Haddad et al., 2016). All these possible explanations may explain the high total yield of tubers in response to potassium at $120 \mathrm{~kg} /$ Feddan regardless the harvesting time in this study. In addition, our study clearly indicated that potassium fertilization has strong effects on the tuber quality, such as tuber dry matter and its bio-chemical compounds, which are important for potato processing (Roe and Faulks, 1991; Kita, 2002). For instance, dry matter contents are related with the contents of starch, proteins and mineral compounds in potato (Naz et al., 2011). Also, the reducing sugars are considered precursors for formation of acrylamide during frying which is neurotoxic and carcinogenic compound (Zorb et al., 2014). On the other hand, the reducing sugars participate in reaction of Maillard, thus produce flavor and color development of potato products. In this study, the potassium fertilization, particularly at 120 $\mathrm{kg} /$ Feddan, significantly enhanced tuber dry matter, total sugars and SSC and reduced non-reducing sugars and total phenolics in both growing seasons. Similarly, it was found that potassium application altered tuber dry matter content and reduced the reducing sugars and total phenolics, leading to an improvement in quality parameters (Westermann et al., 1994; Khan et al., 2010; Homouz et al., 2010; Pervez et al., 2013). This result may be explained by the fact of that potassium stimulates the activity of starch synthase enzyme and consequently convert the simple molecules of glucose into complex starch molecules (Moinuddin et al., 2004). Concerning the effect of potassium on total phenolic content in potato tubers, illustrated results showed that the healthy plants (higher yield) which received $120 \mathrm{~kg} /$ feddan had the lowest phenolic content, while the control plants had the highest values, which may indicate that the control plants might be subjected to stress during the growing seasons. This effect may be attributed to the role of potassium in alleviation of abiotic stress (Cakmak, 2005; Amanullah and Irfanullah, 2016).

Potato has high yielding potential per unit area and it is being soil-exhausting crop, therefore, it needs high organic and inorganic nutrients. However, it is potassium preferring vegetable crop; therefore, it absorbs huge potassium quantities during the growth season (Bishwoyog and Swarnima, 2016; Haddad et al., 2016). One of the significant findings of the current study that mineral composition of potato tubers $(\mathrm{N}, \mathrm{P}$ and $\mathrm{K}$ ) were significantly affected by potassium fertilization in both growing seasons. Briefly, the highest content of phosphorus and potassium in tubers were recorded with the potassium application at 120 $\mathrm{kg}$. These findings are in agreement with results of Eleiwa et al. (2010) and Khan et al. (2012). The high concentration of mineral composition of tubers may be referred to increasing nutrients availability in the soil due to the potassium abundance (Marschner, 1995), or/and due to the potassium role in activation of more than sixty enzymes, which are involved in several biochemical processes in plant cell such as nitrogen metabolism, starch synthesis, respiration and energy utilization (Wallingford, 1980).

One of the interesting outputs of this research is that not all potassium levels has a promotional effect on the vegetative traits, yield attributes, bio-chemical constitutes and mineral composition. This study convincingly indicates that highest level of potassium $(150 \mathrm{~kg})$ has low positive, neutral and even negative effect compared to control, depending on the trait. Similarly, Zelelw et al. (2017) reported that potassium application of more than $150 \mathrm{~kg} \mathrm{~K} 2 \mathrm{O} / \mathrm{ha}$ might be excessive dose that causes decline in tuber production; however, they concluded that this effect is depending on the variety. Generally, several drastic consequences of excess of potassium have been confirmed, such as nitrogen, magnesium and calcium deficiency symptoms, which are directly related to plant growth and productivity (Prajapati and Modi, 2012; Ramyabharathi et al., 2014). Thus, the observed reduction in most of the studied traits might indicate that the potato plants that were fertilized by $150 \mathrm{~kg}$ might be subjected to macro and/or micronutrient deficiency during the growth in response, causing this reduction.

The result of this study indicated that there was a slight variation between the two harvesting times in most of vegetative growth traits, biochemical constitutes and mineral composition over all potassium levels. However, this difference was absent in terms of yield parameters. Briefly, harvesting at 105 days after planting was found to be more responsive and high yielding compared to harvesting at 90 days but the difference was marginal and could be ignored. Zelelw et al. (2017) confirmed that maturity of the potato varieties was gradually delayed with increasing potassium levels, which partially contradict our results. They discussed that potassium is promoting nutrient 
absorption capacity especially $\mathrm{N}$, which delays the initiation of the storage organs and decreases the accumulation of photosynthate in tubers (Gunasena and Harris, 1971; Noor, 2010). However, they have applied high levels of $300 \mathrm{~kg} \mathrm{~K} \mathrm{~K}_{2} \mathrm{O}$, which is more than two times of highest level in our study. Nevertheless, potassium supplementation at high dose (150 kg) makes a reduction in most of the traits in this study, which partially confirms the results of Zelelw et al. (2017). With regard to the effect of potassium application on earliness of potato tubers, the indicated restlts showed that the increasing level of potassium from $90 \mathrm{~kg} /$ feddan to $120 \mathrm{~kg} /$ Feddan produced early potato tubers with full maturity, 15 days early than that only received $90 \mathrm{~kg} /$ Feddan, which needed more time to achieve the maturity stage. The explanation for that may be due to the critical level (120 kg /Feddan) which is needed for increasing translocation of carbohydrates from leaves to tubers. However, increasing the potassium level from 105 to $150 \mathrm{~kg}$ Feddan reduced marketable and total yield, which might be attributed to the toxicity of this level.

\section{CONCLUSION}

Early maturity of potato tubers was associated with high rate of potassium $(120 \mathrm{~kg})$; however, the over dose of potassium $(150 \mathrm{~kg})$ achieved a negative effect compared to the other potassium levels, leading to a significant reduction in the most of the yield traits. Nevertheless, further investigations should follow this study to confirm its results, especially in late season potato cultivars.

\section{REFERENCES}

Abd El-Latif K, M., E. A.M. Osmana, R. Abdullahb and N. Abd el Kader (2011). Response of potato plants to potassium fertilizer rates and soil moisture deficit. Adv. Appl. Sci. Res., 2(2): 388-397.

Amanullah, A. I., and Z. H. Irfanullah (2016). Potassium management for improving growth and grain yield of maize (Zea mays L.) under moisture stress condition. Scientific reports, 6 .

Baethgen, W.E. and M.M. Alley (1989). A manual colorimetric procedure for measuring ammonium nitrogen in soil and plant kjeldahl digests. Comm Soil Sci Plant Anal 20(9\&10):961-969.

Bishwoyog, B., and K. C. Swarnima (2016). Effect of potassium on quality and yield of potato tubers-a review. International Journal of Agriculture \& Environmental Science, 3, 914.

Cakmak, I. (2005). The role of potassium in alleviating detrimental effects of abiotic stresses in plants. J. Plant Nutr. Soil Sci., 168: 521530.

Cutcliffe, J. A. and D. C. Munro (1976). Effects of nitrogen, phosphorus and potassium on yield and maturity of cauliflower. Canadian journal of plant science, 56(1), 127-131.

Dampney, P., S. Wale and A. Sinclair (2011). Review Potash Requirements of Potatoes. Report of Agriculture \& Horticulture Development Board 2011.

Dubois, M. K. A., J. K. Hamilton, P. A. Rebers and F. Smith (1956) Colorimetric method for determination of sugars and related substances. Anal. Chem., 28: 350-356.

Eleiwa, E.M., S. A. Ibrahim and F. M. Mohamed (2012). Combined effect of NPK levels and foliar nutritional compounds on growth and yield parameters of potato plants (Solanum tuberosum L.). African Journal of Microbiology Research. 6(24): 5100-5109.

FAO (Food and Agriculture Organization of the United Nations), (2017) Retrieved December (2019) from the FAOSTAT on the world Wide Web: http://www.fao.org/faostat/en/\#data/QC

Gunasena, H.P.M. and P.M. Harris (1971). The effect of CCC, Nitrogen and Potassium on Growth and Yield on Two Varieties of Potato. The Journal of Agricultural Science, 76, 33-52

Haddad, M., N.M. Bani-Hani, J. A., Al-Tabbal and A.H. Al-Fraihat (2016). Effect of different potassium nitrate levels on yield and quality of potato tubers. Journal of Food, Agriculture \& Environment 14 (1): 101-107.

Havlin, J.L., J.D. Beaton, S.L. Tisdale and W.L. Nelson (2005). Soil Fertility and Fertilizers: An Introduction to Nutrient Management. 7th Edition, Pearson Educational, Inc., Upper Saddle River, New Jersey.

Jackson, M. L. (1973). Soil Chemical Analysis Prentice Halla of India Private Limited. New Delhi, Indian.

Khan M.Z., M. E. Akhtar, M. Mahmood-ul-Hassan, M. M. Mahmood and M. N. Safdar (2012). Potato tuber yield and quality as affected by rates and sources of potassium fertilizer. Journal of Plant Nutrition, 35:664-677.

Khan, M.Z., M.E. Akhtar, M.N. Safdar, M.M. Mahmood, S. Ahmad and N. Ahmed (2010). Effect of source and level of potash on yield and quality of potato tubers. Pak. J. Bot. 42: 3137-3145.

Kita, A. (2002). The influence of potato chemical composition on crisp texture. Food Chem. 76: 173-176.

Liljeroth, E., Å. Lankinen, L. Wiik, D. D. Burra, E., Alexandersson, and E. Andreasson (2016). Potassium phosphate combined with reduced doses of fungicides provides efficient protection against potato late blight in largescale field trials. Crop Protection, 86, 42-55.

Mahmoud, A.R. and M.M. Hafez (2010). Increasing Productivity of Potato Plants (Solanum tuberosum L.) by Using Potassium Fertilizer and Humic Acid Application. International Journal of Academic Research, 2, 83-88. 
Marschner, H. (1995). Mineral Nutrition of Higher Plants. $2^{\text {nd }}$ edn. Academic Press, London, $889 \mathrm{p}$.

Marschner, P. (2012). Mineral Nutrition of Higher Plants, $3^{\text {rd }}$ ed.; Academic Press: London, UK; pp. 178-189.

Mazumdar, B. C. and Majumder K. (2003). Methods on physico-chemical analysis of fruits. Daya publishing house.

Mengel, K. and E.A. Kirkby (2001). Potassium. In Principles of Plant Nutrition (Norwell, MA, USA: Kluwer Academic Publishers), pp. 503- 509 .

Michalska, A., A. Wojdyło and B. Bogucka (2016). The influence of nitrogen and potassium fertilisation on the content of polyphenolic compounds and antioxidant capacity of coloured potato. Journal of Food Composition and Analysis, 47, 69-75.

Moinuddin, K. Singh, S.K. Bansal and N.S. Pasricha (2004). Influence of graded levels of potassium fertilizer on growth, yield and economic parameters of potato. Journal of Plant Nutrition, 27(2): 239-259.

Müller-Röber, B., J. Ellenberg, N. Provart, L. Willmitzer, H. Busch, D. Becker and R. Hedrich (1995). Cloning and electrophysiological analysis of KST1, an inward rectifying $\mathrm{K}^{+}$channel expressed in potato guard cells. The EMBO Journal, 14(11): 2409-2416.

Navarre, D.A., A. Goyer and R. Shakya (2009). Nutritional value of potatoes: phytonutrient and mineral content, pp. 395-424. In: Singh, J. and L. Kaur (eds.). Advances in Potato chemistry and technology. Academic Press, New York.

Naz, F., A. Ali, Z. Iqbal, N. Akhtar, S. Asghar and B. Ahmad (2011). Effect of different levels of NPK fertilizers on the proximate composition of potato crop at Abbottabad. Sarhad Journal of Agriculture, 27(3), 353-356.

Noor, M.A. 2010. Physiomorphological determination of potato crop regulated by potassium management. PhD. Thesis submitted to Institute of Horticultural Sciences University of Agriculture, Faislabad, Pakistan.

Oyarburo, N. S., M. F. Machinandiarena, M. L. Feldman, G. R. Daleo, A. B. Andreu and F. P. Olivieri (2015). Potassium phosphite increases tolerance to UV-B in potato. Plant Physiology and Biochemistry, 88: 1-8.

Page, A.L., R. H. Miller and D. R. Keeney (1982). Methods of Soil Analysis. Part 2: Chemical and Microbiological Properties. ASA, Madison, WI.

Pervez, M.A., C.M. Ayyub, M.R. Shabeen and M.A. Noor (2013). Determination of physiomorphological characteristics of potato crop regulated by potassium management. Pakistan Journal of Agricultural Sciences, 50, 611-615.

Pettigrew, W. T. (2003). Relationships between insufficient potassium and crop maturity in cotton. Agronomy Journal, 95(5): 13231329.

Prajapati, K. and H. A. Modi (2012). The importance of potassium in plant growth-a review. Indian Journal of Plant Sciences, 1(02-03), 177-186.

Radwan, E.A. and Z. S. A. El-Shall (2011). Effect of potassium fertilization and humic acid application on plant growth and productivity of potato plants under clay soil. J. Plant Production, Mansoura Univ., 2 (7): 877- 890.

Roe M. and R. Faulks (1991). Color development in a model system during frying - role of individual amino-acids and sugars. Journal of Food Science 56: 1711-1713.

Sadasivam, S. and A. Manickam (1991). Biochemical methods for agricultural sciences. Wiley Eastern Limited, New Dellhi and Tamil Nadu Agricultural University, Coimbatore, pp. 5-6.

Saha, R., S. Mondal and J. Das (2001). Effect of potassium with and without sulfur containing fertilizers on growth and yield of potato (Solanum tuberosum L.). Environment and Ecology, 19(1), 202-205.

Wallingford, W. (1980). Function of potassium in plants In: Potassium for Agriculture. Potash and Phosphate Inst., Atlanta. Georgia., pp: 10-27.

Westermann, D.T., D.W. James, T.A. Tindal, and R.L. Hurst (1994). Nitrogen and potassium fertilization of potatoes: sugars and starch. Am. Potato J. 71: 433453.

Zekri, M. and T.A. Obreza (2009). Plant nutrients for citrus trees. SL,200, UF\IFAS Extension Service, Institute of Food and Agricultural Sciences, University of Florida.

Zelelew, D.Z., S. Lal, T.T. Kidane and B.M.G. Ghebreslassie (2016). Effect of potassium levels on growth and productivity of potato varieties. American Journal of Plant Sciences, 7(12): 1629-1638.

Zorb C., M. Senbayram and E. Peiter (2014). Potassium in agriculture - Status and perspectives. Journal of plant physiology 171(9): 656-669. 


\section{الإنتاج المبكر للبطاطس تأثر إيجابياً بالتسميد البوتاسي}

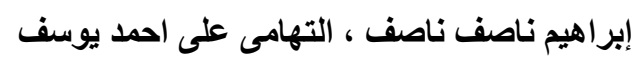

قسم البساتين، كلية الزر اعة، جامعة قناة السويس، الإسماعيلية لئل

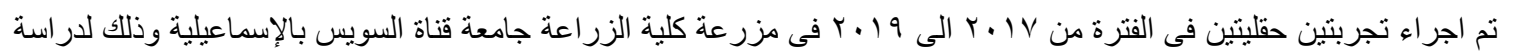

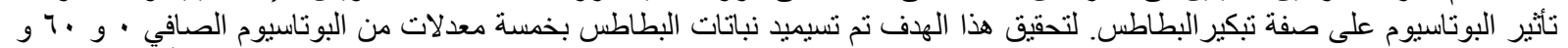

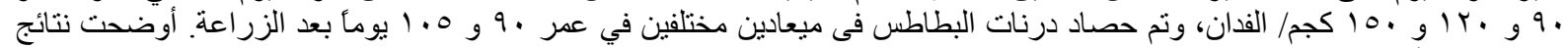

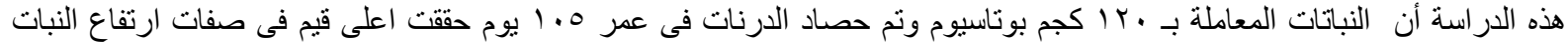

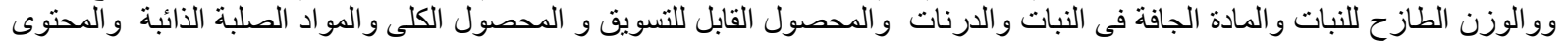

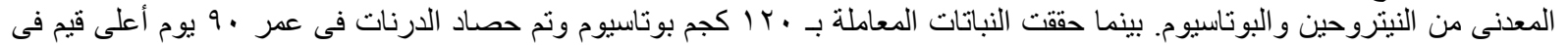

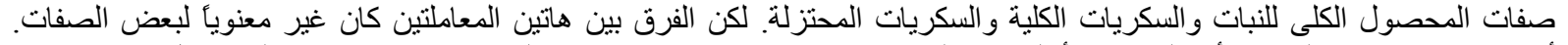

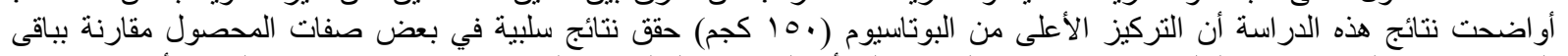

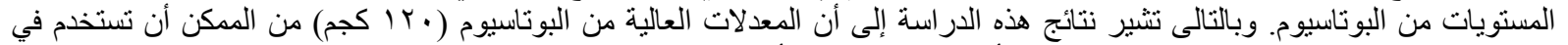

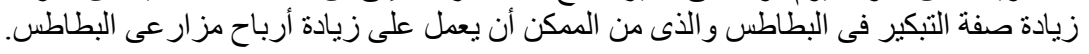

